\title{
The Research Progress of SiRNA Targeting Notch1 on Tumor Cells: A Mini Review of the State of the Art
}

\author{
Lanfen $\mathrm{Huo}^{1}$; Shaoling $\mathrm{Wu}^{1 *}$; Zhonghai $\mathrm{Chi}^{2}$; Xindong $\mathrm{Zhao}^{3}$ \\ ${ }^{1}$ Department of Hematology, Affiliated Hospital of Qingdao University, Qingdao, Shandong, P.R. China \\ ${ }^{2}$ Hospitals of Traditional Chinese and Western Medicine, Cangzhou city, Hebei, P.R. China \\ ${ }^{3}$ Department of Hematology, Medical College of Qingdao University, Qingdao, Shandong, P.R. China
}

\begin{abstract}
Notch signaling is a highly conserved signaling pathway, playing an important role in a variety of cell differentiation, development and regulation. Notch signaling includes Notch1-4; Notch1 gene encodes Notch1 signaling that can shorten cell cycle, enhance cell proliferation, inhibit cell differentiation, and promote apoptosis. Mutation and overexpression of the Notch1 gene may induce tumorigenesis, which plays an important role in the development of tumors across a variety of signaling pathways. Currently, using RNA interference technology (RNAi) synthesizing small interference RNA (siRNA) targeting Notch1 gene (siNotch1) has become a hot topic, and clinical application of gene silencing has also obtained a certain therapeutic effect. In this paper, the application of Notch1 gene silencing in tumor progress was reviewed. (Int J Biomed. 2016;6(3):163-166.).
\end{abstract}

Key Words: RNAi • Notch signaling • Notch1 gene • tumor cells.

\section{Introduction}

The Notch signaling pathway includes the Notch receptor proteins, Notch ligand proteins, transcription factors CSL (DNA binding protein), and target molecules. In mammals, four Notch genes (Notch1-4) and at least five of their ligands (Jagged1 and 2; Delta1, 3, and 4) are identified. ${ }^{[1]}$ Notch1 is one of the Notch signaling pathways, by the Notch1 gene regulation. The Notch1 gene can shorten the cell cycle, quicken cell proliferation, inhibit cell differentiation and promote apoptosis. ${ }^{[2]}$ The Notch1 gene, in any link in the process of T-cell development disorder, is likely to be associated with cell malignant transformation. ${ }^{[3]}$

Pancewicz J et al. ${ }^{[4]}$ have found activating mutations in Notch in more than $30 \%$ of adult T-cell leukemia (ATL) patients. Activated Notch signaling contributes to $\sim 50 \%$ of human T-cell acute lymphoblastic leukemia (T-ALL) cases through gain-offunction mutations in the Notch1 gene. ${ }^{[5]}$ Therefore, Notch1 gene mutation and high expression may induce the occurrence of hematological malignancies and play an important role in a broad range of tumor occurrences and development. ${ }^{[6,7]}$

Corresponding authors: Prof. Shaoling Wu, Department of Hematology, Affiliated Hospital of Qingdao University, Qingdao, Shandong, P.R. China; E-mail: qdwushaoling@163.com

\section{The siRNA targeting the Notch1 gene in normal cells}

The Notch signaling pathway has been shown to regulate angiogenesis and endothelial cell formation, and is found in numerous cell types. Increased Notch signaling in endothelial stalk cells correlates with vessel regression, whereas reduced Notch signaling in the stalk leads to formation of a new tip cell. ${ }^{[8]}$

Recent studies revealed crucial roles of the Notch system in mature $\mathrm{T}$ cell differentiation and activation. Notch1 plays an obligatory and selective role in $\mathrm{T}$ cell lineage induction. Notch1 was also reported to modulate the $\beta$ selection step of thymocytes. There are a number of articles suggesting that $\gamma \delta /$ $\alpha \beta$ T-cell fate decisions are modulated by Notch signaling. In addition, it promotes the $\mathrm{CD} 4+, \mathrm{CD} 8+$ double positive thymus cells to differentiate into single positive thymus cells. ${ }^{[9]}$ It has been suggested that Notch1 signaling plays an important role in the process of mature T-lymphocytes, and Notch signaling disorders may lead to the occurrence of T-cell leukemia or lymphoma.

\section{The siRNA targeting the Notch1 gene in tumor cells}




\subsection{Respiratory system}

Hassan et al. ${ }^{[10]}$ found that Notch1 has an inhibitory tumor function, especially in the context of switching off cell invasion and metastasis. Notch1 signaling in small cell lung carcinoma (SCLC) controls H69AR and SBC3 cell adhesion and epithelial mesenchymal transition (EMT). Overexpression of Notch1 in SCLC switched off EMT, cell motility and cell metastatic potential.

\subsection{Digestive system}

Sun et al. ${ }^{[11]}$ studied the Notch1 pathway and the VEGF pathway with human gastric cancer cells (SGC7901) and found the siJagged1 affected SGC7901 cell proliferation and apoptosis by acting on Notch1 and VEGF signaling pathways.

Notch signaling pathway has been reported to play critical roles in hepatocellular carcinoma (HCC). Liu Hong showed that transfection of Notch1 small-interfering RNA (siRNA) into nude mice HCC cells resulted in cell growth inhibition and apoptosis. ${ }^{[12]}$ Notch signaling was found to positively regulate cell proliferation in hepatoma HepG2 cell lines and GSI treatment inhibited tumor cell proliferation through the suppression of Notch signaling.

In recent years, studies have reported that Notch1 gene silencing has a therapeutic effect on pancreatic cancer cells, ${ }^{[13]}$ which offers a new direction for the treatment of pancreatic cancer.

\subsection{Urinary system}

Zhang et al. ${ }^{[14]}$ studied Notch1 expression in renal cell carcinoma and silence the expression of Notch1 by using siRNA and observed its effect on the proliferation of Caki- 1 . To detect expression of Notch1 mRNA by RT-PCR and Notch1 protein levels by Western blot, siRNA interference transfected into RCC Caki-1 cells, transfection with Lipofectamine 2000 for 48 hours. Notch1 in renal cell carcinoma was high expressed compared with normal renal tubular epithelial cells. Specific siRNA interference significantly reduced the Caki-1 cells, mRNA and protein expression, and Caki-1 cells proliferation was significantly decreased. These results suggest that siRNAmediated silencing of the Notch-1 gene may represent a novel target for gene therapy of renal cancer cells.

Ai Xing et al. ${ }^{[15]}$ found that Notch1 gene expression increased in bladder cancer cell line BIU87 and that the interference of Notch1 gene expression had a therapeutic effect on bladder cancer cells. In addition, studies have found that the proliferation capacity of BIU 87 was reduced after using siNotch1. This result may be associated with Notch1 signaling pathway suppressing and influencing the G1 stage of cell differentiation. ${ }^{[16]}$

\subsection{Reproductive system}

Notch1 was found to be overexpressed in prostate cancer (PCa) cells and human PCa tissue. Bin Hafeez et al. ${ }^{[17]}$ showed that small interfering RNA-mediated knockdown of
Notch1 in PC3 and 22Rnu1 PCa cells dramatically decreased their invasion. ${ }^{[17]}$ Wang et al. ${ }^{[18]}$ found that down-regulation of Notch-1 and Jagged-1 could inhibit cancer cell migration and invasion, which was in part due to down-regulation of NF-kB and its downstream target genes such as MMP-9, uPA, and VEGF. From these results, authors concluded that down-regulation of Notch-1 or Jagged-1 could potentially be an effective therapeutic approach for the inactivation MMP9, uPA, and VEGF, which is likely to result in the inhibition of cell growth, migration, invasion and metastasis of prostate cancer.

Notch1 gene expression in cervical cancer tissue is higher than in normal cervical tissue. Some studies have found that the Notch1 gene has a significant positive correlation with tumor differentiation, and that the Ki67 gene is a sign of cell proliferation. The expression of the Notch1 gene has a positive correlation with Ki67 in cervical cancer tissue. ${ }^{[19]}$ This suggests that Notch1 signaling is involved in cell proliferation in cervical cancer tissues.

\subsection{Blood system}

Weng et al. ${ }^{[5]}$ reported that more than $50 \%$ of human T-ALLs, including tumors from all major molecular oncogenic subtypes, have activating mutations that involve the extracellular heterodimerization domain and/or the C-terminal PEST domain of Notch-1. These findings greatly expand the role of activated Notch-1 in the molecular pathogenesis of human T-ALL. Thus, siNotch1 can be used as a new method for treatment of T-ALL.

Yang et al. ${ }^{[20]}$ researched the therapeutic effect of siRNA of proton-sponge-coated quantum dots (QD) on Notch1 protein over expression in T-ALL cell line. and found that the gene silencing efficiency of proton-sponge-coated QD-siRNA increased by 4-10 folds, and proton-sponge-coated QD-siRNA also decreased the content of Notch1 mRNA significantly, as compared with controls. This illustrates that proton-spongecoated QD-siRNA can be used for treatment of T-ALL.

Weng et al. ${ }^{[2]}$ studied T-ALL and found that c-MYC is a direct downstream target of Notch1 in Notch-dependent T-ALL cell lines, that contributes to the growth of T-ALL cells.. The existence of a direct link between Notch and c-MYC in T-ALL cell lines and normal thymocytes has therapeutic as well as basic implications. The c-MYC gene as a potential Notch1 target provides new ideas and a new direction for the application of siNotch1 in clinical treatment of leukemia.

Kamstrup et al ${ }^{[22]}$ studied the Notch 1 gene as a potential target for cutaneous T-cell lymphoma treatments. Specific down-regulation of Notch1 by siRNA induced apoptosis in SeAx. The caspase 3/7 activity increased significantly in the Notch1 siRNA-transfected group compared with the control siRNA-transfected group 24 hours after transfection. Seventytwo hours after transfection, the apoptosis rate was up to peak. The mechanism of apoptosis involved the inhibition of $\mathrm{NF}-\mathrm{kB}$, which is the most important prosurvival pathway in cutaneous T-cell lymphoma. These data show that Notch is present in cutaneous T-cell lymphoma and that its inhibition may provide a new way to treat cutaneous T-cell lymphoma. ${ }^{[22]}$ 


\subsection{Others}

In human brain glioma cells, Notch1 gene mRNA and protein expression are significantly higher than in non-tumor cells. ${ }^{[23]} \mathrm{Xu}$ Peng et al. ${ }^{[24]}$ studied Notch1 expression in the majority of 45 astrocytic gliomas with different grades and in U251MG glioma cells. Transfection of siRNA targeting Notch1 into U251 cells in vitro downregulated Notch1 expression, associated with inhibition of cell growth, arrest of cell cycle, reduction of cell invasiveness, and induction of cell apoptosis. Meanwhile, tumor growth was delayed in established subcutaneous gliomas in nude mice treated with Notch1 siRNA in vivo. These findings suggest that Notch1 plays an important oncogenic role in the development and progression of astrocytic gliomas; siNotch1 is expected to be a promising direction for the treatment of astrocytic gliomas. Other studies have reported that the Notch1 gene also plays a part in the development of breast cancer. ${ }^{[25,26]}$

\section{The clinical application of RNA interference}

Davis et al. ${ }^{[27]}$ conducted the first in-human phase I clinical trial involving the systemic administration of siRNA to patients with solid cancers using a targeted, nanoparticle delivery system (clinical version denoted as CALAA-01). They provided the actual evidence of inducing an RNAi mechanism of action in a human from the delivered siRNA. siRNA administered systemically to a human can produce a specific gene inhibition (reduction in mRNA andprotein) by an RNAi mechanism of action.

Schultheis et al ${ }^{[28]}$ evaluated the safety, tolerability, and pharmacokinetics of Atu027 (a Liposomal siRNA inhibitor for PKN3) in a first-in-human phase I study and found that Atu027 was safe in patients with advanced solid tumors, with $41 \%$ of patients having stable disease for at least 8 weeks. It is expected to become a new method for the treatment of solid tumors.

\section{Conclusion and prospect}

Above all, Notch1 signaling plays an important role in the regulation of cell proliferation, differentiation, apoptosis, and invasion. Although siNotch1-related research in the field of tumor cytology has made some progress, it is mostly in the basic research stage at present; its specific mechanism is relatively limited and not comprehensive enough. Achieving its clinical application specification is also facing many difficulties and challenges, such as the problems of designing siRNA segments, effective transfection, off-target effects, delivery system, etc. At the same time, security may deserve attention. In addition, how Notch1 can be applied directly to the human body to interfere with the growth of tumor cells is a problem that has to be solved, and to do so requires further research.

In sum, further study of the mechanism by which Notch1 and siNotch1 interact with tumor cells is necessary at the molecular and gene, protein and animal model levels.
For the future, a technique called RNA interference will be widely used in biomedical research. We believe that with the deepening of the research on gene silencing, siRNA targeting the Notch1 gene is expected to become the new tool for cancer treatment.

\section{Competing interests}

The authors declare that they have no competing interests.

\section{References}

1. Artavanis-Tsakonas S, Rand MD, Lake RJ. Notch signaling: cell fate control and signal integration in development. Science. 1999; 284(5415):770-6.

2. Cullion K, Draheim KM, Hermance N, Tammam J, Sharma VM, Ware C, et al. Targeting the Notch1 and mTOR pathways in a mouse T-ALL model. Blood. 2009; 113(24): 6172-81.

3. Jundt F, Anagnostopoulos I, Forster R, Mathas S, Stein $\mathrm{H}$, Dorken. Activated Notch1 signaling promotes tumor cell proliferation and survival in Hodgkin and anaplastic large cell lymphoma. Blood. 2002; 99(9): 3398-403.

4. Pancewicz J, Taylor JM, Datta A, Baydoun HH, Waldmann TA, Hermine $\mathrm{O}$, Nicot $\mathrm{C}$. Notch signaling contributes to proliferation and tumor formation of human T-cell leukemia virus type 1-associated adult T-cell leukemia. Proc Natl Acad Sci USA. 2010; 107(38):16619-24.

5. Weng AP, Ferranndo AA, Lee W, Morris JP 4th, Silverman LB, Sanchez-lrizarry C, et al. Activating mutations of NOTCH1 in human T cell acute lymphoblastic leukemia. Science. 2004; 306(5694): 269-71.

6. Wang XX, Wu SL, Zhao XD, Liu XP, Chang C. The expression and signifiance of Notch1 protein in lymphoma. Med J QiLu. 2013; 28(1):8-10.

7. Li GH, Huang SY, Kang ZJ, Xu H, Liang YM. Research advance of notch signal in ex vivo expansion of hematopoietic progenitor cells - review. Zhongguo Shi Yan Xue Ye Xue Za Zhi. 2008;16(5):1227-31. [Article in Chinese].

8. Phng LK, Potente M, Leslie JD, Babbage J, Nyqvist D, Lobov I, et al. Nrarp coordinates endothelial Notch and Wnt signaling to control vessel density in angiogenesis. Dev Cell. 2009; 16(1): 70-82.

9. Tsukumo S, Yasutomo K. Notch governing mature T cell differentiation. J Immunol. 2004;173(12):7109-13.

10. Hassan WA, Yoshida R, Kudoh S, Hassegawa K, NiimoriKita K, Ito T. Notch1 controls cell invasion and metastasis in small cell lung carcinoma cell lines. Lung Cancer. 2014; 86(3): 304-10.

11. Sun HW, Wu C, Tan HY, Wang QS. Combination DLL4 with Jagged1-siRNA can enhance inhibition of the proliferation and invasiveness activity of human gastric carcinoma by Notch1/VEGF pathway. Hepatogastroenterology. 2012; 59(115):924-9.

12. Liu H. Effects of siRNA targeting Notch1 on the proliferation of and apoptosis in xenografted human hepatocellular carcinoma cells in nude mice. China Journal of Modern Medicine. 2012; 22(4):15-19.

13. Du X, Wang YH, Wang ZQ, Cheng Z, Li Y, Hu JK, et al. Down-regulation of Notch1 by small interfering RNA enhances chemosensitivity to gemcitabine in pancreatic cancer cells through activating apoptosis activity. Zhejiang Da Xue Xue Bao Yi Xue Ban. 2014; 43(3):313-8. [Article in Chinese]. 
14. Zhang Z, Wang K, Cao ZL, Hao ZQ, Guo FF. Effect of interference of Notch1 by siRNA on proliferation in renal cell carcinoma Caki-1 cells. Journal of Modern Oncology. 2014; 22(2):292-5.

15. Ai X, Wu Z, Shi TP, Liu SL, Wang C, Zhang GX, et al. The influence of shRNA-induced Notch1 gene silencing on the biological behavior of bladder cancer cell line BIU87. Journal of Clinical Urology. 2009; 24(5):389-92.

16. Wang Z, Zhang Y, Li Y, Banerjee S, Liao J, Sarkar FH. Down-regulation of Notch-1 contributes to cell growth inhibition and apoptosis in pancreatic cancer cells. Mol Cancer Ther. 2006; 5(3):483-93.

17. Bin Hafeez B, Adhami VM, Asim M, Siddiqui IA, Bhat KM, Zhong W, et al. Targeted knockdown of Notch1 inhibits invasion of human prostate cancer cells concomitant with inhibition of matrix metalloproteinase- 9 and urokinase plasminogen activator. Clin Cancer Res. 2009; 15(2):452-9.

18. Wang Z, Li Y, Banerjee S, Kong D, Ahmad A, Nogueira $\mathrm{V}$, et al. Down-regulation of Notch-1 and Jagged-1 inhibits prostate cancer cell growth, migration and invasion, and induces apoptosis via inactivation of Akt, mTOR, and NF- $\mathrm{BB}$ signaling pathways. J Cell Biochem. 2010; 109(4):726-36.

19. Sun Y, Zhang R, Zhou S, Ji Y. Overexpression of Notch1 is associated with the progression of cervical cancer. Oncol Lett. 2015; 9(6): 2750-6.

20. Yang YP, Li W, Lin H, Du ZH, Wang GJ. Therapeutic effects of proton-sponge-coated QD-siRNA targeting Notch1 protein on leukemia. Journal of Immunology. 2013; 29(06): 474-79. [Article in Chinese].

21. Weng AP, Millholland JM, Yashiro-Ohtani Y, Arcangeli
ML, Lau A, Wai C, et al. c-Myc is an important direct target of Notch1 in T-cell acute lymphoblastic leukemia/lymphoma. Genes Dev. 2006; 20(15):2096-109.

22. Kamstrup MR, Gjerdrum LM, Biskup E, Lauenborg BT, Ralfkiaer E, Woetmann A, et al. Notch1 as a potential therapeutic target in cutaneous T-cell lymphoma. Blood. 2010; 116(14): 2504-12.

23. Purow BW, Haque RM, Noel MW, Su Q, Burdick MJ, Lee $\mathrm{J}$, et al. Expression of Notch-1 and its ligands, Delta-like-1 and Jagged-1, is critical for glioma cell survival and proliferation. Cancer Res. 2005; 65(6): 2353-63.

24. Xu P, Qiu M, Zhang Z, Kang R, Jiang R, Jia z, et al. The oncogenic roles of Notch1 in astrocytic gliomas in vitro and in vivo. J Neurooncol. 2010; 97(1):41-51.

25. Mao J, Mou QJ, Li LH, Tao YJ, Fan ZJ, Wang B, et al. Effect on proliferation and apoptosis of human breast cancer line MCF-7 with Notch1 gene silencing by RNA interference. J Clin Exp Pathol. 2012; 28(01):11-4.

26. Reedijk M, Odorcic S, Chang L, Zhang H, Miller N, McCready DR, et al. High-level coexpression of JAG1 and NOTCH1 is observed in human breast cancer and is associated with poor overall survival. Cancer Res. 2005; 65(18): 8530-7. 27. Davis ME, Zuckerman JE, Choi CH, Seligson D, Tolcher A, Alabi CA, et al. Evidence of RNAi in humans from systemically administered siRNA via targeted nanoparticles. Nature. 2010; 464(7291):1067-70.

28. Schultheis B, Strumberg D, Santel A, Vank C, Gebhardt F, Keil O, et al. First-in-human phase I study of the liposomal RNA interference therapeutic Atu027 in patients with advanced solid tumors. J Clin Oncol. 2014; 32(36):4141-8. 